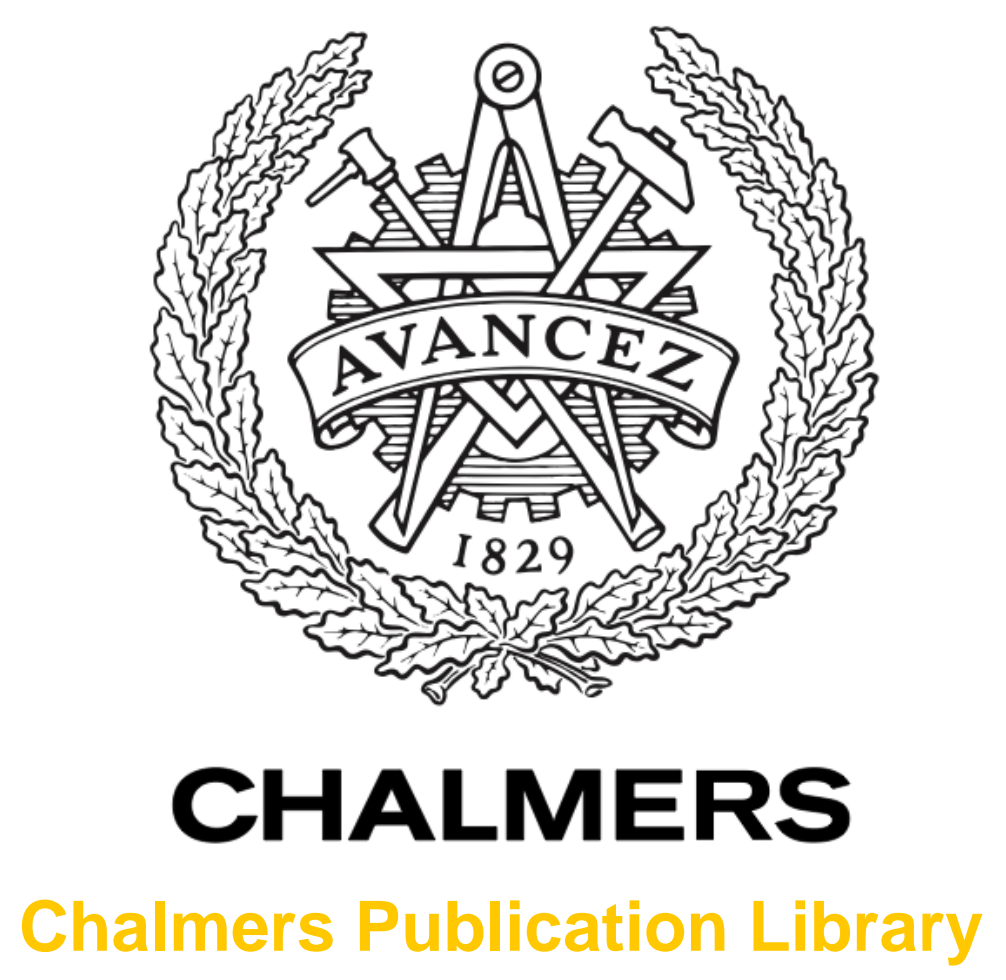

\title{
Several new ultra-wideband antenna systems for radio telescopes and industry sensor imaging process
}

This document has been downloaded from Chalmers Publication Library (CPL). It is the author's version of a work that was accepted for publication in:

2012 14th International Conference on Electromagnetics in Advanced Applications, ICEAA 2012. Cape Town, 2 - 7 September 2012

Citation for the published paper:

Yang, J. ; Yin, J. ; Pantaleev, M. (2012) "Several new ultra-wideband antenna systems for radio telescopes and industry sensor imaging process". 2012 14th International Conference on Electromagnetics in Advanced Applications, ICEAA 2012. Cape Town, 2 - 7 September 2012 pp. 1281-1284.

http://dx.doi.org/10.1109/ICEAA.2012.6328825

Downloaded from: http://publications.lib.chalmers.se/publication/163452

Notice: Changes introduced as a result of publishing processes such as copy-editing and formatting may not be reflected in this document. For a definitive version of this work, please refer to the published source. Please note that access to the published version might require a subscription.

Chalmers Publication Library (CPL) offers the possibility of retrieving research publications produced at Chalmers University of Technology. It covers all types of publications: articles, dissertations, licentiate theses, masters theses, conference papers, reports etc. Since 2006 it is the official tool for Chalmers official publication statistics. To ensure that Chalmers research results are disseminated as widely as possible, an Open Access Policy has been adopted.

The CPL service is administrated and maintained by Chalmers Library. 


\title{
Several New Ultra-wideband Antenna Systems for Radio Telescopes and Industry Sensor Imaging Process
}

\author{
Jian Yang ${ }^{1}$, Jungang Yin ${ }^{2}$, Miroslav Pantaleev ${ }^{3}$, Yinan Yu ${ }^{1}$, Tomas Mc Kelvey ${ }^{1}$, Seyedeh S. \\ Fayazi ${ }^{1}$, Hoi-Shun Lui ${ }^{1}$
}

\begin{abstract}
This paper presents an overview of several ultrawideband (UWB) antenna systems recently developed at Chalmers for applications in future UWB radio telescopes and industry sensor imaging process.
\end{abstract}

\section{INTRODUCTION}

Due to its quite unique properties, ultra-wideband (UWB) technology finds many applications in different areas, such as UWB sensor network for precise ranging and geolocation, UWB radar and imaging systems with superior penetration and high resolution, and super sensitive UWB radio astronomy.

In the paper, we present an overview of several new UWB antenna systems developed at Antenna Group at Chalmers University of Technology, including the circular Eleven feed reflector antenna system [1] for future UWB radio telescopes, such as the square kilometer array (SKA) and very long baseline interferometer (VLBI2010), a UWB indoor radar system [2], and a UWB antenna sensor imaging system for industry product processing [3].

\section{The circular Eleven feed for reflector antennas for radio telescopes}

Figure 1 shows the modeling and prototype of the new developed circular Eleven feed. It is known that only $n=1$ component (referred to as $\mathrm{BOR}_{1}$ component) in the Fourier series of the far-field function of a feed contributes to the directivity of the reflector antenna, while all other $n \neq 1$ components cause sidelobes. Therefore, a high $\mathrm{BOR}_{1}$ efficiency (the power in BOR1 component relative to the total radiated power) is critical for achieving a high aperture efficiency and low sidelobes in feed design.

One way for a feed to achieve a high $\mathrm{BOR}_{1}$ efficiency is to make its geometry more rotationally symmetrical. Figure 2 shows the aperture efficiency and its sub-efficiencies of the circular Eleven feed when it illuminates a reflector with a subtended angle of $2 \times 60^{\circ}$, based on the simulated and the measured radiation function. Very good agreement between the simulation and the measurement is observed. The aperture efficiency is higher than $60 \%$ over $1-10$ $\mathrm{GHz}$ and $50 \%$ up to $14 \mathrm{GHz}$, which is a significant improvement, especially below $2.2 \mathrm{GHz}$ and above $13 \mathrm{GHz}$, compared to the previous $2-13 \mathrm{GHz}$ Eleven feed consisting of straight folded dipoles in [4]. The simulated and measured reflection coefficient is shown in Figure 3.

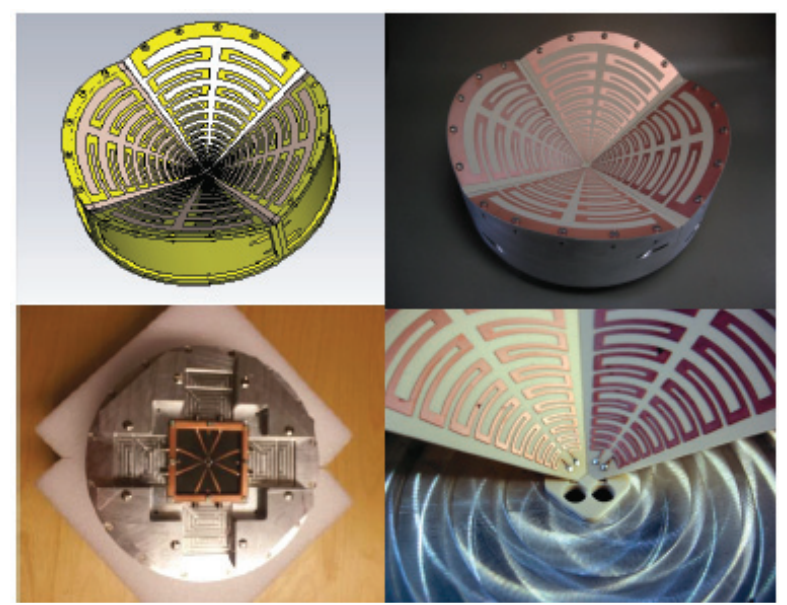

Figure 1: The circular Eleven antenna with dual linear polarization, with the diameter and the height of $210 \mathrm{~mm}$ and $65 \mathrm{~mm}$, respectively: (left-up) simulation model; (right-up) proto-type; (left-down) center puck on rear side; (rightdown) detailed center puck on front side.

We have investigated the effect of the cryostat used in our VLBI2010 project on the radiation performance by CST simulation. The simulation model and the calculated aperture efficiency are shown in Figure 4. Compared to the aperture efficiency without the cryostat in Figure 2, we can see that the cryostat helps to improve the spillover efficiency at the low frequencies, and has no effect on the performance at high frequencies.

\footnotetext{
${ }^{1}$ Dept. Signals and Systems, Chalmers University of Technology, 42196 Goteborg, Sweden,

e-mail: jian.yang,yinan.yu, tomas.mckelvey, antony.lui@chalmers.se, fayazi@student.chalmers.se.

${ }^{2}$ Dept. Electronics and Telecommunications, Norwegian Univ. of Science and Technology (NTNU), Norway,

e-mail: jungang.yin@iet.ntnu.no.

${ }^{3}$ Dept. Earth and Space Science, Chalmers Universuty of Technology, 42196 Goteborg, Sweden,

e-mail: miroslav.pantaleev@chalmers.se.
} 

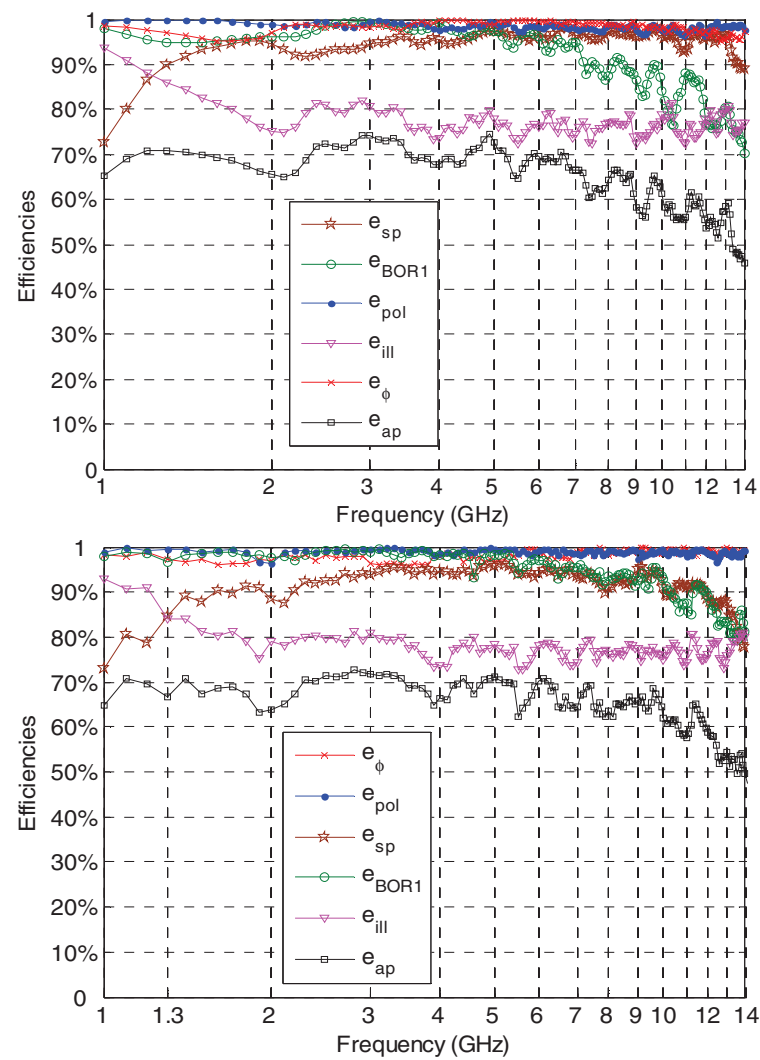

Figure 2: Calculated aperture efficiency and its subefficiencies when the circular feed illuminates a paraboloid with a subtended angle of $2 \times 60^{\circ}$, based on (up) simulated and (down) measured radiation function.

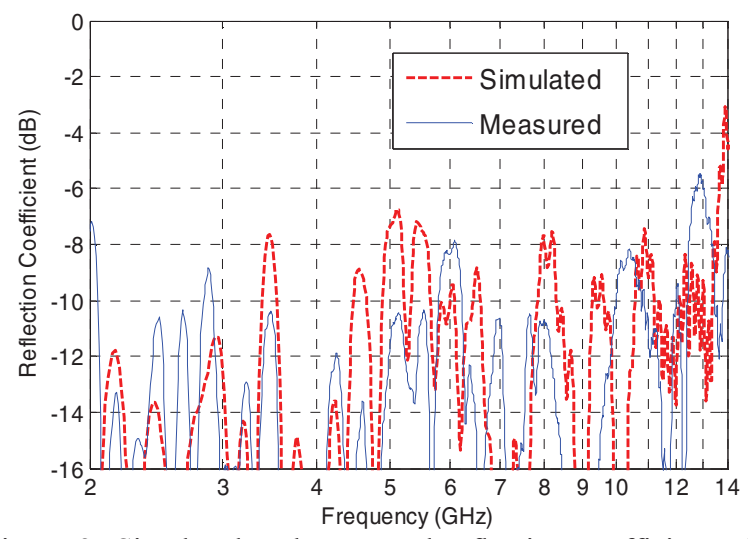

Figure 3: Simulated and measured reflection coefficient of the circular Eleven feed.

\section{A Compact UWB Indoor Radar System}

UWB radar systems exhibit several distinct advantages over the narrowband counterparts: 1) higher ranging resolution with higher penetration ability through lossy materials simultaneously; 2) enhanced target recognition; 3) better penetration ability to passive interference (i.e., rain, fog, clutter); 4) very low cost.

A new compact and low-cost UWB radar system for ranging and tracking of moving objects in indoor and

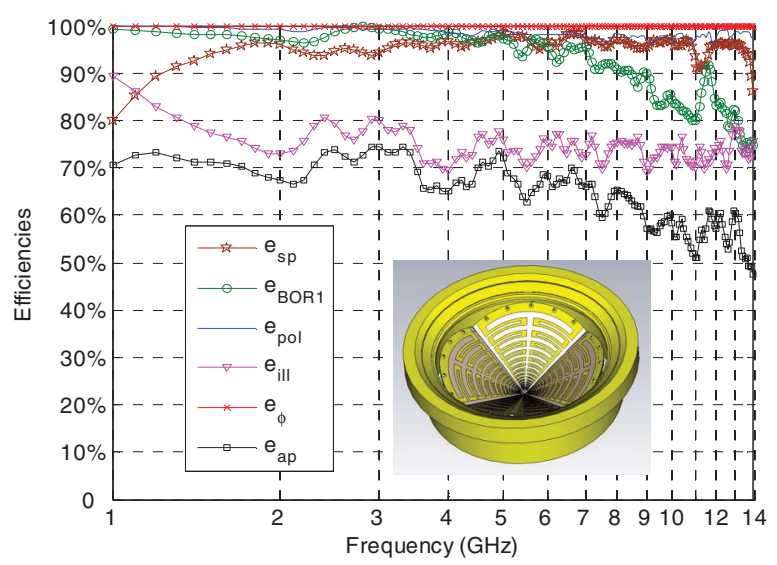

Figure 4: Calculated aperture efficiency and its subefficiencies when the circular feed located inside the cryostat illuminates a paraboloid with a subtended angle of $2 \times 60^{\circ}$, based on simulated radiation function.

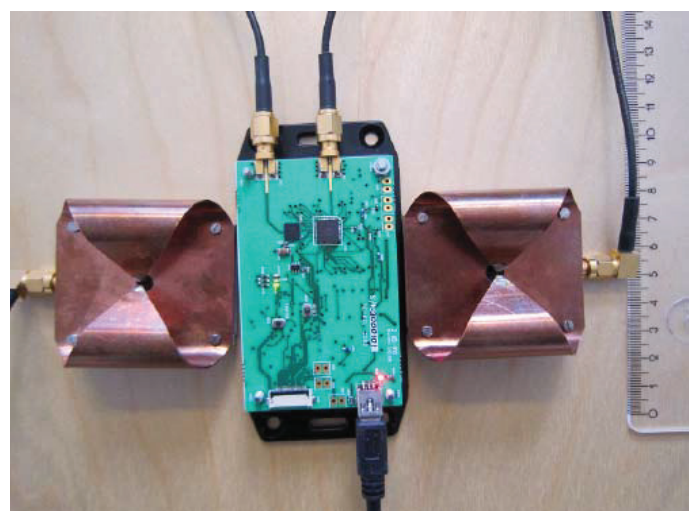

Figure 5: The compact UWB radar system for indoor and through-wall ranging and tracking.

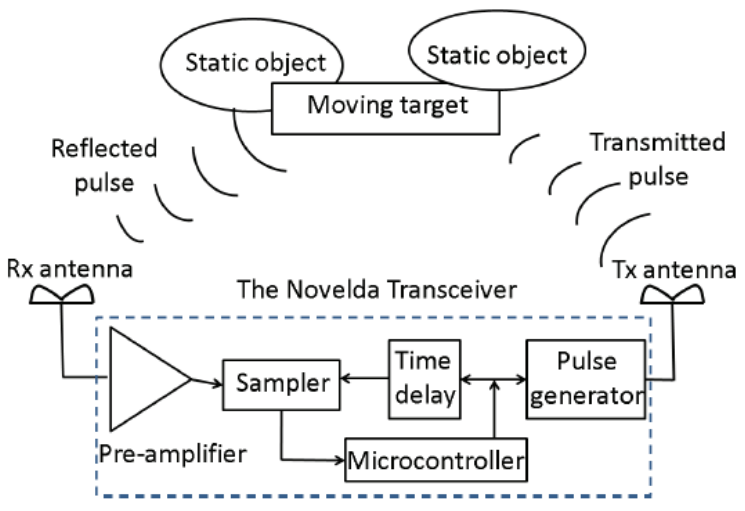

Figure 6: Operating principle of the compact UWB radar system

through-wall environments has been developed, see Figure 5. The UWB radar consists of a commercially available Novelda R2A UWB CMOS chip transceiver operating over 2-8 GHz and the recently developed UWB 2-15-GHz self-grounded Bow-Tie antennas [5]. Pulses are transmitted via one antenna (Tx antenna), 
reflected by targets, and then received by the second antenna (Rx antenna), as depicted in Figure 6.

The measured time-domain response of the UWB radar system is shown in Figure 7 , when the two antennas are separated by a distance of $250 \mathrm{~mm}$ in the face-to-face configuration in an anechoic chamber. From the figure, two time-response measures, the width of the pulse full-width at halfmaximum (FWHM) $\tau_{\mathrm{FWHM}}$ and the duration of the ringing $\tau_{\mathrm{r}=0.22}$, can be obtained as $\tau_{\mathrm{FWHM}}=134 \mathrm{ps}$ and $\tau_{\mathrm{r}=0.22}=260 \mathrm{ps}$, which states a fast time pulse response of the radar system. Please refer to [6] for the definitions of $\tau_{\mathrm{FWHM}}$ and $\tau_{\mathrm{r}=0.22}$.

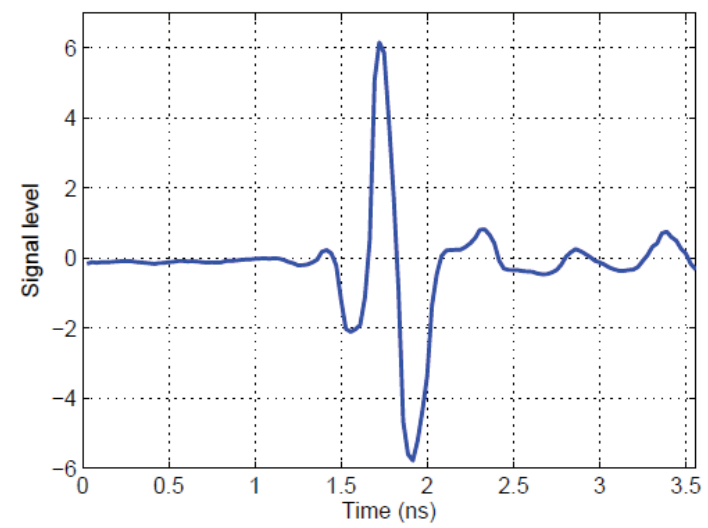

Figure 7: Measured time-domain impulse response of the UWB radar system when the two antennas are separated by $250 \mathrm{~mm}$ in face-to-face configuration.

\begin{tabular}{|c|c|c|}
\hline Algorithm & $\begin{array}{c}\text { Without } \\
\text { clutter map } \\
\text { removal }\end{array}$ & $\begin{array}{r}\text { With clutter } \\
\text { map removal }\end{array}$ \\
\hline $\begin{array}{c}\text { Pulse signature } \\
\text { matching }\end{array}$ & $75.4(\mathrm{~mm})$ & $48.1(\mathrm{~mm})$ \\
\hline $\begin{array}{c}\text { Pulse-spectrum } \\
\text { signature matching }\end{array}$ & $7.2(\mathrm{~mm})$ & $2.6(\mathrm{~mm})$ \\
\hline $\begin{array}{c}\text { Pulse-spectrum } \\
\text { signature matching } \\
\text { subsample delay } \\
\text { estimation }\end{array}$ & $5.0(\mathrm{~mm})$ & $1.4(\mathrm{~mm})$ \\
\hline
\end{tabular}

Tabel 1: Comparison of the ranging techniques in terms of RMSE between the real and estimated positions.

A robust and accurate algorithm for ranging and tracking, the pulse-spectrum signature matching plus subsample delay estimation, have been developed [2]. The ranging function of the radar system is evaluated by the measurements of a metal ball with a diameter of $20 \mathrm{~mm}$ moving in an indoor environment. Tabel 1 shows the ranging accuracy for this case by different algorithms. It can be seen that the ranging accuracy achieves to $1-2 \mathrm{~mm}$. Figure 8 illustrates the tracking performance of the radar system, where the red dot in Matlab program screen follows the movement of the target very well.

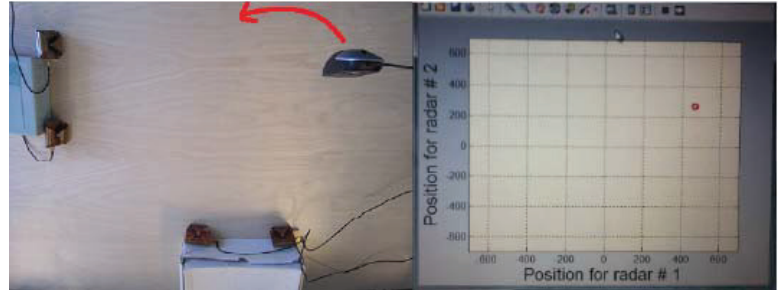

Figure 8: An orthogonal measurement setup for two dimensional tracking (left), and the graphical user interface implemented in Matlab (right), where the red dot indicates the location of the target.

\section{Imaging of Near-Field Object Using UWB SAR Algorithm}

UWB technology and its use in imaging and sensing have drawn significant interest in the last two decades. Extensive studies have contributed to utilizing UWB transient scattering for automated target recognition and imaging purposes [7], [8], where a UWB synthetic aperture radar (SAR) imaging algorithm was presented and applied for subsurface object imaging. One limitation of the algorithm is that an artefact in the imaging procedure causes ambiguity. Here, a simple modified procedure is proposed to reduce such artefact. The feasibility of the proposed algorithm is presented and verified using experimental data.

The UWB imaging system for objects in near-field region is shown in Figure 9. It consists of a selfgrounded Bow-Tie antenna located in front of the object, a vertically placed rectangular metal plate. To obtain multiple views, the metal plate is placed in 68 equal-spaced positions along a straight line of 1088 $\mathrm{mm}$ long, and the monostatic responses at each of these 68 positions are measured using a network vector analyzer (VNA) from $0.5 \mathrm{GHz}$ to $13 \mathrm{GHz}$. The above setup is equivalent to the case that a single antenna moves along a straight line in front of the static object, and transmits and receives the monostatic responses at these 68 positions, as shown in Figure 10. Therefore, the SAR algorithm can be employed to have an imaging of the object.

The image is reconstructed by the simple SAR, shown in Figure 11(up). The vertically-placed metal plate is observed but it comes with a "smile" shape around it. This smiling is an undesirable artefact introduced from the algorithm. To remove this artefact, we use the same antenna moving in the other side of the object, say behind the object. The same measurement and image reconstruction procedures are repeated. The resultant image is shown in Figure 11 (down). It is observed that the "smile" is changed to a "crying" in the image. By a correlation of these two images - each pixel intensities of the two images are multiplied by each other, the "smile" and the "crying" are canceled while the object image remains. The resultant image is shown in Figure 12 
and it is clear that the artefact has been significantly suppressed.

\section{Conclusions}

An overview of several UWB antenna systems is presented to show the applications of new developed UWB systems in different areas.

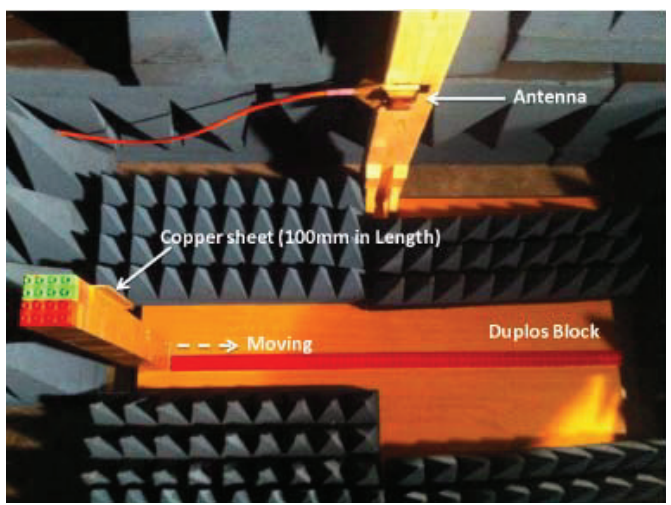

Figure 9: The test UWB imaging system

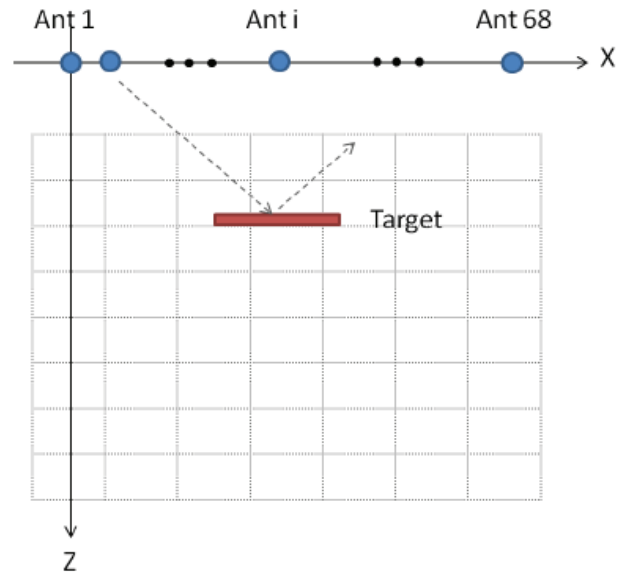

Figure 10: The geometry of the SAR configuration

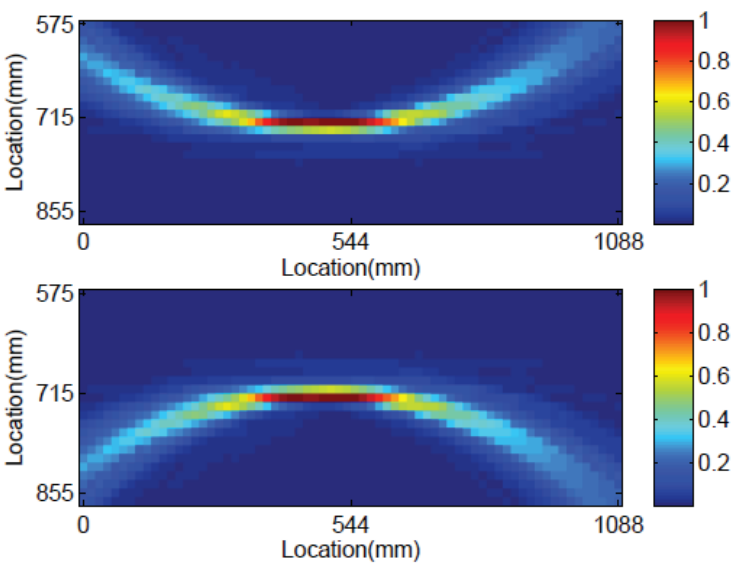

Figure 11: Image reconstruction results based on: (a) front antenna (up); (b) back antenna (down).

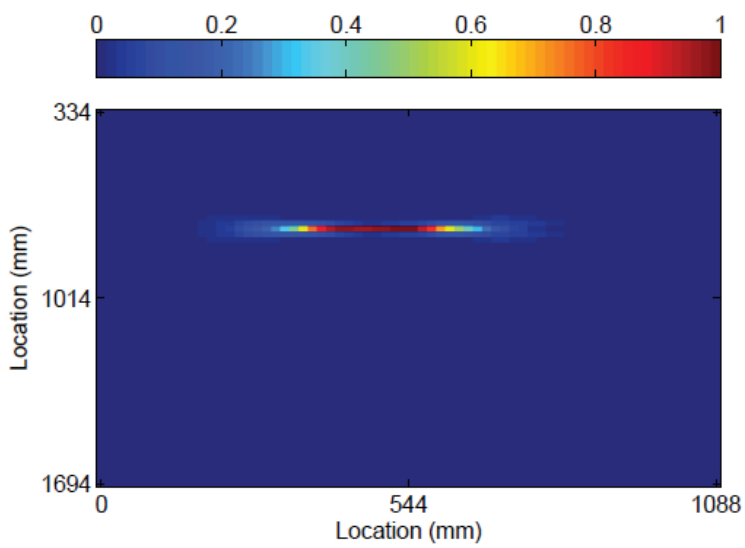

Figure 12: The resultant image after modified algorithm.

\section{References}

[1] J. Yin, J. Yang, M. Pantaleev, L. Helldner, "The circular Eleven antenna: A new Decadebandwidth feed for reflector antennas with a high aperture efficiency", submitted to IEEE Trans. Ant. Propag., April, 2012.

[2] Y. Yu, J. Yang, T, McKelvey and B. Stoew, "A compact UWB indoor and through-Wwll radar with precise ranging and tracking", International Journal Ant. Propag., July, 2012.

[3] S. S. Fayazi, H.-S. Lui and J. Yang, "Microwave imaging of near-field object using ultraWideband synthetic aperture radar algorithm", 2012 IEEE International Symposium on Antennas and Propagation July 8-14, 2012, Chicago, USA.

[4] J. Yang, M. Pantaleev, P.-S. Kildal, B. Klein, Y. Karadikar, L. Helldner, N. Wade-falk, and C. Beaudoin, "Cryogenic 2-13 GHz Eleven feed for reflector antennas in future wideband radio telescopes," IEEE Trans. Antennas Propagat., vol. 59, no. 6, pp. 1918-1934, June 2011.

[5] J. Yang and A. Kishk, "A novel low-profile compact directional ultra-wideband antenna: the selfgrounded Bow-Tie antenna," IEEE Trans. Antennas Propagat., vol. 60, no. 3, pp. 1214-1220, March 2012.

[6] W. Wiesbeck, G. Adamiuk, and C. Sturm, "Basic properties and design principles of UWB antennas," Proceedings of the IEEE, vol. 97, no. 2, pp. 372-385, 2009.

[7] Y. Wang, I. D. Longstaff, and C. J. Leat, "SAR Imaging of Buried Objects from MoM modelled scattered field," IEE Proc. Radar, Sonar and Navigation, vol. 148, no. 3, pp. 167-172, 2001.

[8] S. Vitebskiy, L. Carin, M. A. Ressler, and F. H. Le, "Ultra-Wideband, Short-Pulse GroundPenetrating Radar: Simulation and Measurement," IEEE Trans. Geoscience and Remote Sensing, vol. 33, no. 3, pp. 762-772, 1997. 